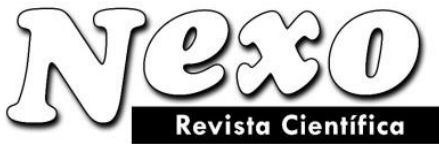

ISSN-E 1995-9516

Universidad Nacional de Ingeniería COPYRIGHT @ (UNI). TODOS LOS DERECHOS RESERVADOS

http://revistas.uni.edu.ni/index.php/Nexo

https://doi.org/10.5377/nexo.v34i04.12638

\title{
Dinámica de procesos de innovación educativa mediante el análisis de redes sociales
}

\section{Dynamics of educational innovation processes through the analysis of social networks}

\author{
Patricia del C. Lacayo-Cruz * \\ Universidad Nacional de Ingeniería. Facultad de Ciencias y Sistemas. Managua, Nicaragua. \\ "patricia.lacayo@fcys.uni.edu.ni
}

(recibido/received: 16-junio-2021; aceptado/accepted: 08-agosto-2021)

\begin{abstract}
RESUMEN
Se analiza la temática de procesos de innovación educativa, mediante el Análisis de Redes Sociales (ARS) a fin de determinar las aristas de los procesos de innovación educativa que realizan los docentes de la carrera de Ingeniería de Sistemas de la Universidad Nacional de Ingeniería (UNI) con su respectivo orden, en el I Semestre del año 2021. Encontrando que, "documentar" es el proceso de innovación educativa que menos se realiza y el de mayor práctica es "medición de resultado", además se identifican los procesos que tienen oportunidad de mejora en futuras estrategias de capacitación relacionadas con la innovación educativa en la carrera.
\end{abstract}

Palabras claves: Investigación Educativa, Métrica Gephi, Capacitación Docente, Redes de Procesos.

\begin{abstract}
The subject of educational innovation processes is analyzed, through the Analysis of Social Networks (ARS) in order to determine the edges of the educational innovation processes carried out by teachers of the Systems Engineering career of the National University of Engineering (UNI) with its respective order, in the I Semester of the year 2021. Finding that, "documenting" is the educational innovation process that is least carried out and the one with the most practice is "measurement of results", in addition, the processes that have the opportunity are identified. improvement in future training strategies related to educational innovation in the career.
\end{abstract}

Keywords: Educational Research, Gephi Metrics, Teacher Training, Process Networks.

\section{INTRODUCCIÓN}

La innovación educativa es un proceso creativo cuyo principal objetivo es mejorar aspectos relacionados con el aprendizaje del alumno, en ese propósito el carácter creativo del proceso de innovación hace difícil 
la planificación, la medición de los resultados, la aplicación eficaz y eficiente y la transferencia de ese proceso creativo (Fidalgo-Blanco \& Sein-Echaluce, 2018).

Otra problema asociada a la innovación educativa, es el conocimiento de un inventario de procesos de innovación educativa que ejecutan los docentes, como parte de un curriculo oculto existente en muchas escuelas y universidades, este curriculo oculto se desarrolla en los planes de estudios y es de gran importantica en la investigación formativa en mejora de las capacidades actitudinales del docente (Benavides, 2018). Además, estos procesos de innovación educativa pueden ser analizados en una red de procesos, ya que existe poca comprension de las aplicaciones de la minería de redes, y su implementación con el mundo real está en gran parte inexplorado (Bonchi et al., 2011).

En este contexto, la Facultad de Ciencias y Sistemas (FCYS) de la Universidad Nacional de Ingeniería (UNI) de Nicaragua no es una excepción a la necesidad del conocimiento de una red procesos de innovación educativa realizado por sus docentes, por lo tanto, es importante preguntarse: ¿Será que el grado medio con pesos en las conexiones de la red permite ubicar los procesos de la innovación educativa que más practican los docentes, en conjunto con la identificación de los procesos que puedan tener oportunidad de mejora en los docentes.?. En respuesta a la anterior pregunta de investigación se requiere determinar las aristas de los procesos de innovación educativa que realizan los docentes de la carrera de Ingeniería de Sistemas de la UNI con su respectivo orden, en el I Semestre del año 2021, que permita la orientación de estrategias en futuras capacitaciones.

El conocer los procesos de innovación educativa que más practicas los docentes de la carrera de Ingeniería de Sistemas, fortalece la opinión de los tomadores de decisiones de la UNI sobre futuras temáticas de capacitación, asimismo la comprensión del orden de ejecución de los procesos de innovación educativa que realizan los docentes de la carrera, puede orientar la estratégia metodológica de las capacitaciones hacia la comprensión de un enfoque de procesos sistémicos de la innovación educativa.

En la siguiente sección se explica el origen de la operacionalización de los procesos de innovación educativa, luego la metodología que expone el uso del Análisis de Redes Sociales (ARS) en la identificación gráfica de los procesos de innovación educativa, por último, se exponen los resultados encontrados con sus respectivas conclusiones.

\section{FUNDAMENTOS TEÓRICOS}

En muchas ocasiones, se tiene la idea que la Innovación Educativa tiene que ver con inventar algo totalmente novedoso respecto al proceso Enseñanza/Aprendizaje, sin embargo, el presente estudio considera la definición de la Innovación Educativa según lo expresado por García-Peñalvo (2015) como:

Realizar cambios en el aprendizaje/formación que produzcan mejoras en los resultados de aprendizaje. Sin embargo, para que se considere innovación educativa el proceso debe responder a unas necesidades, debe ser eficaz y eficiente, además de sostenible en el tiempo y con resultados transferibles más allá del contexto particular donde surgieron (p. 6).

$\mathrm{Al}$ analizar la definición anterior, el primer proceso que se identifica es la aplicación de esos cambios en el aprendizaje/formación que realiza el docente, esa aplicación de la innovación educativa en donde el docente percibe una posible mejora en los resultados de aprendizaje que tendrán sus alumnos, para ello el docente tendrá que medir el resultado del aprendizaje, en esa medición se identifica un segundo proceso el cual es la medición de resultado.

Un tercer proceso identificado es la comparación de esos resultados, producto de la medición previamente realizada. El cuarto proceso que se expone, es la medición de recursos utilizados para que sea eficaz y eficiente. El quinto proceso, se produce en la réplica que generó ese cambio de aprendizaje/formación en 
los alumnos, luego el sexto proceso que sería documentar todo lo realizado hasta el momento para que en último proceso el séptimo, poderlo compartir a otros docentes o entidades.

Esta visión de proceso en la innovación educativa, es justificada por Fidalgo-Blanco \& Sein-Echaluce (2018), los cuales expresan:

Esta situación justifica que se integre la visión de los procesos de ingeniería con la visión de los procesos de innovación educativa (García-Peñalvo, 2015), ya que abre las puertas a que la innovación educativa se pueda planificar, medir, clasificar, organizar y a que, principalmente, tenga un impacto transformador en el modelo educativo. ( $\mathrm{p} 85$ )

Lo planteado por Fidalgo-Blanco \& Sein-Echaluce (2018), crea la posibilidad de identificar los procesos en forma gráfica haciendo uso del Análisis de Redes Sociales (ARS). ARS, es un estudio de la estructura social basado en un método cuantitativo por medio de patrones de comportamiento en una red, esta red se caracteriza por la tenencia de nodos los cuales pueden ser personas, grupos, entidades, objetos, en dependencia del campo de estudio (Kuz et al., 2016).

Este Análisis de Redes Sociales, Kuz et al. (2016) lo explican como un análisis estructural que se ha perfeccionado como herramienta en el estudio de la estructura de las relaciones sociales que afectan la conducta y actitudes de una persona, grupo u objetos, y la influencia de las interacciones que estos pudieran tener en la estructura social de la red.

La lógica del ARS se ha utilizado en: la comprensión de las estrategias de red en Twitter utiliza en la comunicación sobre socios de Responsabilidad Social Empresarial (RSE) de las principales organizaciones sin fines de lucro de los EE. UU (Dong \& Rim , 2019); la investigación de los mecanismos organizativos de aprendizaje entre directivos que conducen al establecimiento de redes de asesoramiento intra organizacionales, que facilitan la adquisición y difusión de conocimientos para la innovación (Ghinoi \& Di Toma, 2021). Entre otros estudios, cuyo fundamento del ARS es la teoría de grafo (Tabassum et al., 2018).

\section{METODOLOGÍA}

En la medición se utilizó un enfoque cuantitativo, con un alcance exploratorio, cuyo método de selección de la muestra fue no probabilístico. "La ventaja de una muestra no probabilística — desde la visión cuantitativa - es su utilidad para determinados diseños de estudio que requieren no tanto una "representatividad" de elementos de una población" sino una cuidadosa y controlada elección" (Hernández Sampieri et al., 2014, p. 190), de docentes que imparten clases en la carrera de Ingeniería de Sistemas, los cuales fueron previamente mencionadas en el planteamiento del problema.

También, se consideró una muestra de 61 docentes de las distintas sedes y programas de la UNI donde se imparten clases de la carrera de Ingeniería de Sistemas, las cuales son: IES (Instituto de Estudios Superiores) en Managua, RUACS (Recito Universitario Augusto Césas Sandino) en Estelí, RURC (Recinto Universitario Región Central) en Juigalpa, PROMECYS (Programa de Modalidad Especial de Ciencias y Sistemas) en Managua y en el RUPAP (Recinto Universitario Pedro Araúz Palacios ) en Managua.

El método utilizado en el estudio fue el ARS para entender la morfología y topología específica de la red de innovación educativa. Además, la técnica de recolección de datos que se utilizó fue un custionario, cuyos datos se recolectaron en formularios de Microsoft Forms 365.

Las preguntas que se utilizaron en el cuestionario están relacionadas a los nodos o procesos relacionados con la Innovación Educativa según definición de García-Peñalvo (2015). A los docentes se les solicitó que seleccionaran en qué orden han realizado los procesos de innovación educativa, además se les indicó que si nunca han realizado algunos de esos procesos que no marcaran orden alguno. En el mismo instructivo, se 
les explicó que deberían entender como Cambios en el Aprendizaje / Formación (C-A/F) a la aplicación de: Diapositivas, Banner, Poster, Video, WebQuest, Guías, entre otros; que implementan en sus asignaturas.

En ese cuestionario, los docentes tenían que marcar en una escala de orden del primer lugar al séptimo lugar los 7 procesos encontrados en la definición de García-Peñalvo (2015), orientados en las siguientes preguntas: 1) ¿Aplica el $\mathrm{C}-\mathrm{A} / \mathrm{F}$ en su grupo de clase, donde halla percibido mejora en los resultados de aprendizaje?; 2) ¿Mide el resultado de aprendizaje antes y después de haberlo aplicado el C-A/F en su grupo de clase?; 3) ¿Compara las mediciones de un momento inicial y un momento final en los resultados de aprendizaje cuando aplica el C-A/F en su grupo de clase?; 4) ¿Mide los recursos utilizados en la aplicación del C-A/F que generó mejora en los resultados de aprendizaje?; 5) ¿Replica el C-A/F que generó mejora en los resultados de aprendizaje, en otros grupos de clase?; 6) Documenta el C-A/F que generó mejoras en los resultados de aprendizaje?; 7) ¿Comparte el C-A/F que generó mejoras en los resultados de aprendizaje con otros docentes?.

En el entendido que ARS es considerado como el estudio de cualquier estructura basado en un método cuantitativo por medio del cual se obtienen estructuras de patrones de relaciones establecidas entre los nodos de una red definidas como grupos (Kuz et al., 2016), se establece que la red de procesos de innovación educativa en estudio contenga 14 nodos, donde los 7 primeros nodos corresponden a los nombres de los procesos de innovación educativa identificados en la definición de García-Peñalvo (2015) relativo a: Aplicación, Medición del resultado, Comparación, Medición de recursos, Replica, Documentación y Compartir. Los últimos 7 nodos corresponden a los lugares según el orden de ejecución de los procesos establecido por los encuetados, etiquetados como: 1erLugar, 2doLugar, 3erLugar, 4toLugar, 5toLugar, 6toLugar y 7 moLugar.

Estos lugares de ubicación de los procesos, son los que el docente encuestado selecciona según el orden en que él ejecuta los procesos de innovación educativa, esta acción crea una arista o enlace entre nodos. Las aristas, son conexiones explícitas que los encuestados declaran dentro de la estructura de la red que indica un enlace entre nodos (Bonchi et al., 2011).

La herramienta de software utilizada es Gephi versión 0.9.2, recurso informático empleado en el gráfico de las aristas y nodos, además en el cálculo de la métrica de grado medio con pesos (número de vecinos conexos a otros nodos) que ocurren en la red de procesos de innovación educativa, pesos de arcos que son proporcionales a un número de diferentes caminos pasando a través de un arco entre dos nodos (Kuz et al., 2016).

Utilizando los resultados obtenidos del grado medio con pesos en la red de procesos de innovación educativa, se propone contrastar la siguiente hipótesis:

H1: Los docentes de la carrera de Ingeniería de Sistemas de la UNI practican la mayor cantidad de procesos de Innovación Educativa.

\section{RESULTADOS}

La red de procesos de innovación educativa en estudio la cual tiene una cantidad de 14 nodos resultó con 383 aristas, por la existencia de aristas paralelas detectadas se utilizó una estrategia de mezclado de suma, lo cual permitió generar una asignación de pesos según la frecuencia de ocurrencia de la arista. El grado medio con pesos resultante de la red de procesos de innovación educativa es de 54.714.

En cuanto a los docentes que participaron en la encuesta, el $21.3 \%$ le corresponde al IES, en igual porcentaje a los procedentes del PROMECYS y del RUPAP, el 23\% al RUACS y el 13.1\% al RURC. En lo cual se aprecia, una aproximación de porcentaje de participación equivalentes en cada Sede y Programa en donde se imparte la carrera de Ingeniería de Sistemas en la UNI. 
Al calcular la Modularidad de la red en la detección de comunidades con el algoritmo de Blondel et al. (2008), se generó una Modularidad de 0.117 y 3 comunidades que se agrupan en la red, cuyas comunidades se visualizan en la Figura 1, donde se aprecia que la clase de modularidad 2 contiene la máxima cantidad de nodos y la clase de modularidad 1 contiene únicamente 3 nodos.

\section{Size Distribution}

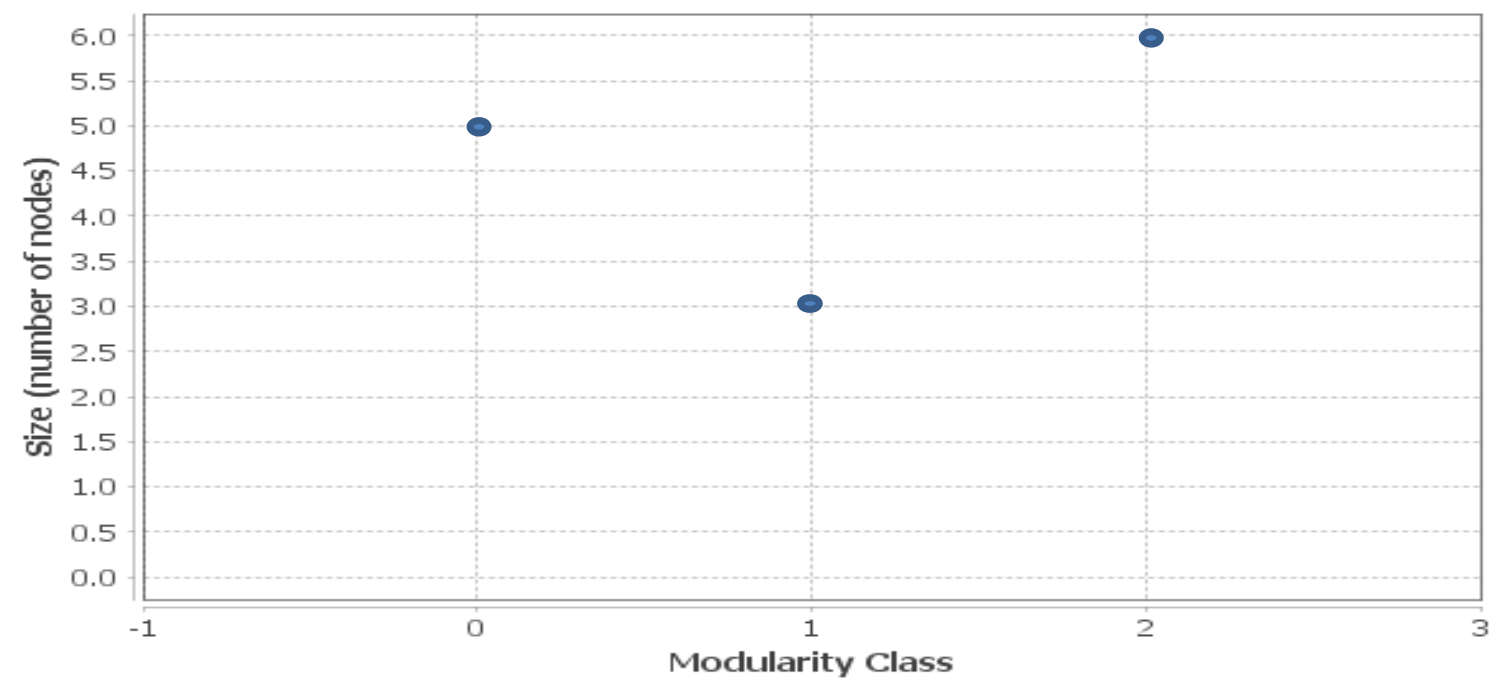

Figura 1. Diagrama de Modularidad de la red de procesos de innovación educativa

El grafo tiene una longitud media de camino de 1.484, lo cual indica una distancia media de nodo a nodo (también conocida como longitud media de camino más corto) (Kuz et al., 2016). Además, posee una cercanía de distribución de centralidad máxima de 12 nodos y mínima de 2 nodos, como se muestran en la Figura 2. Asimismo, distribución de centralidad intermedia mínima de 2 nodos y máxima de 6 nodos. La cercanía se puede considerar como una medida de cuánto tiempo llevará difundir la información de un nodo a todos los demás nodos de forma secuencial. La centralidad de intermediación cuantifica el número de veces que un nodo actúa como puente a lo largo del camino más corto entre otros dos nodos (Kuz et al., 2016).

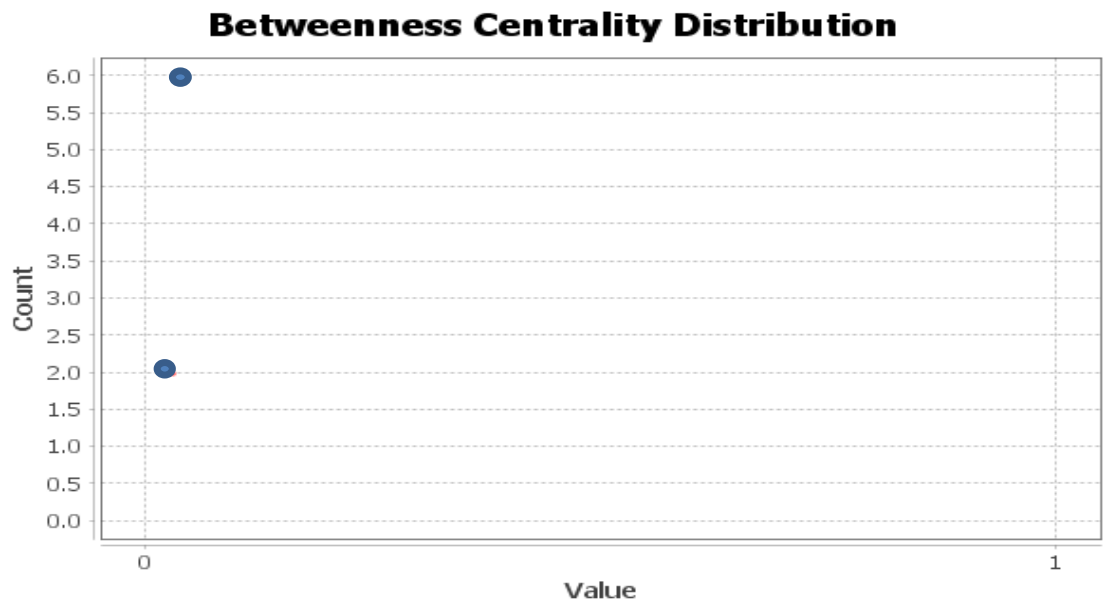




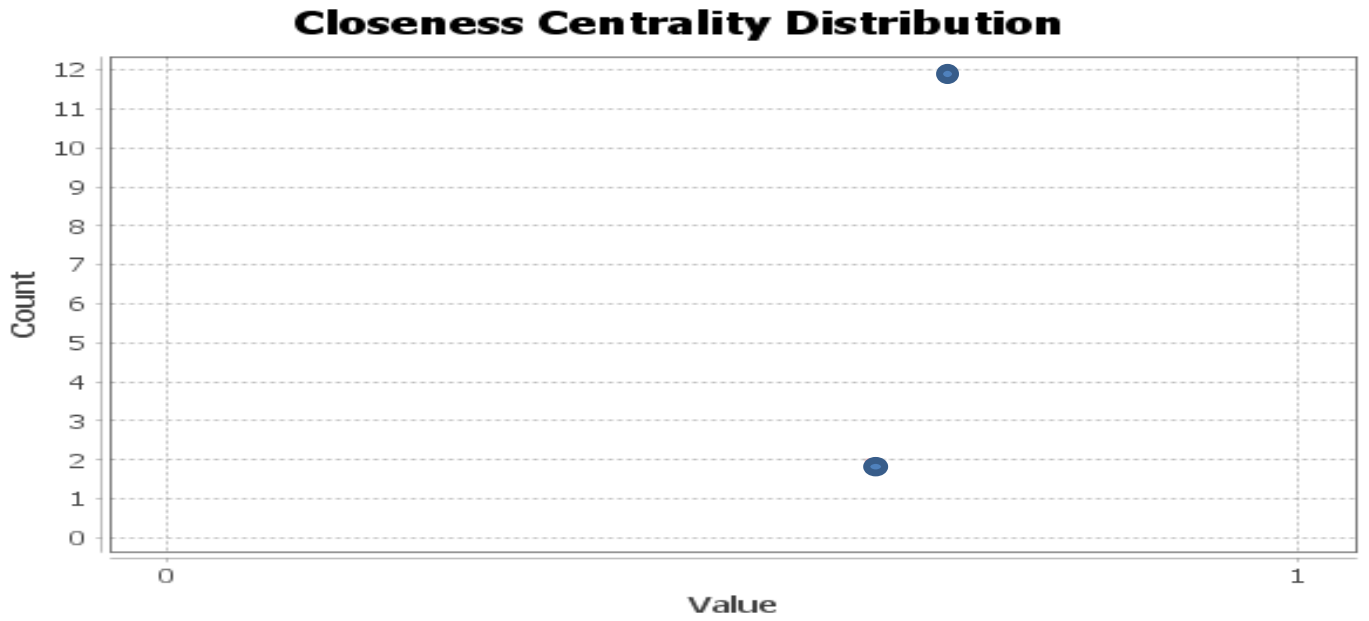

Figura 2. Diagramas de Distribución de Centralidad

El la Figura 3 se observa el grafo de la red de procesos de innovación educativa, donde el tamaño y la intensidad de los nodos está orientado al grado medio con pesos, indicando el número de aristas que pasan por esos nodos. Los grados medios con pesos resultantes en los procesos de innovación educativa, se mencionan ordenados de mayor a menor, Medición de resultado 98, Aplicación 54, Réplica 50, Comparación 47, Medición de recursos 46, Compartir 46, Documentación 42. Asimismo, los grados medios con pesos resultantes del orden de ejecución de los procesos se destacan ordenados de mayor a menor, 1erLugar 127, 2doLugar 89, 3erLugar 55, 4toLugar 51, 7moLugar 32, 5toLugar 12 y 6toLugar 11.

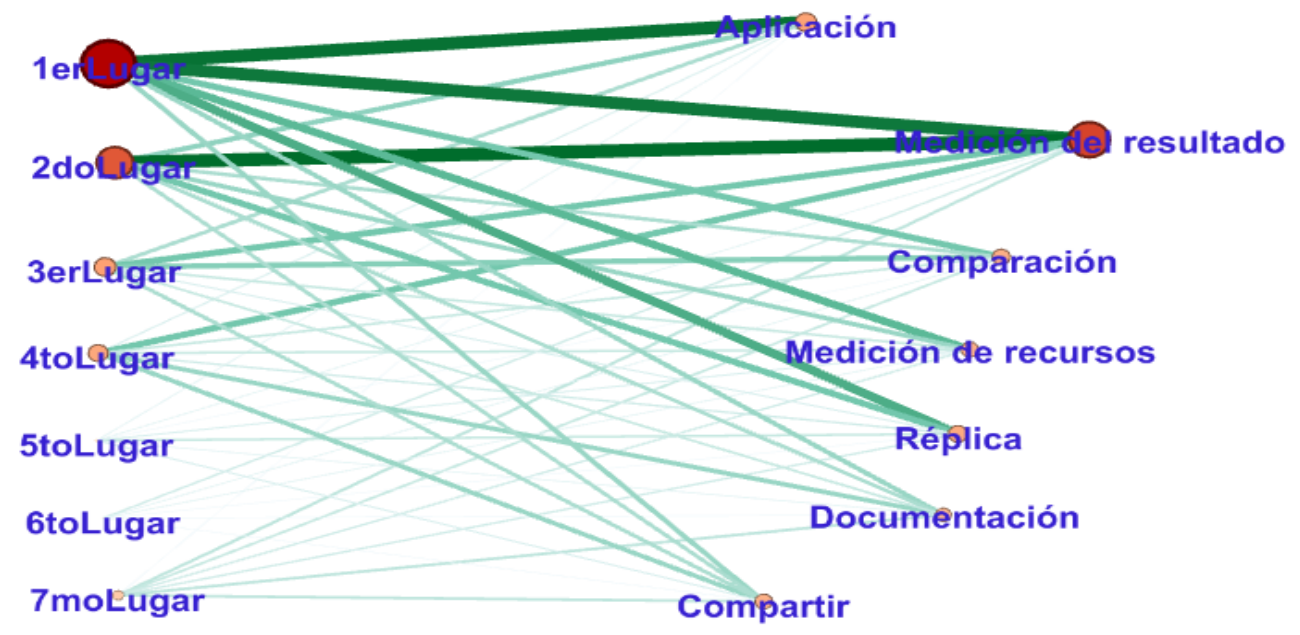

Figura 3. Grafo de la Red de Procesos de Innovación Educativa en docentes de la carrera de Ingeniería de Sistemas de la Universidad Nacional de Ingeniería.

\section{CONCLUSIONES}

Es importante notar que, de todos los procesos de innovación educativa presentado a los docentes, Documentar es lo que menos realizan, lo que más realizan es el proceso de Medición de resultado y 
Aplicación, esto último rechaza la hipótesis que los docentes de la carrera de Ingeniería de Sistemas de la UNI practican la mayor cantidad de procesos de Innovación Educativa. Además, se observa que el proceso que tiene mayor grado medio con pesos es la Medición de resultado, es posible que algunos encuestados midan los resultados de la innovación educativa de forma natural, sin darse cuenta que aplican innovación educativa. También, el mayor grado medio con pesos observado en el orden de los procesos de innovación educativa, apunta al 1erLugar, alude a que algunos encuestados tienen poca percepción de un orden en los procesos de innovación y por lo tanto quieran hacer todos los procesos al mismo tiempo. Conjuntamente, los procesos que tienen mayor oportunidad de mejora en los contenidos temáticos de capacitación institucional relativo a la práctica de la innovación educativa en docentes de la carrera de Ingeniería de Sistemas en la UNI son: Comparación, Medición de recursos, Réplica, Documentación y Compartir la experiencia de innovación educativa.

Este resultado, referente al tema: Procesos de Innovación Educativa realizados por los docentes de la carrera de Ingeniería de Sistemas de la Universidad Nacional de Ingeniería (UNI) de Nicaragua, en el II trimestre del año 2021; está en línea con los hallazgos previos de Zhang et al. (2019) en su artículo titulado: "Social Network Analysis of Sustainable Human Resource Management from the Employee Training's Perspective"; en el sentido que, el análisis de redes sociales funciona bien para estudiar los comportamientos de capacitación de los empleados, facilitando el análisis de similitudes en los diferentes tipos de conductas formativas en capacitaciones de recursos humanos.

Una oportunidad de mejora del presente estudio sería ampliar la muestra en todas las carreras de la UNI con todos los docentes en sus distintas modalidades, que permita una mejor toma de decisión o estrategia a seguir en los procesos de capacitación institucional y no solo a nivel de carrera.

\section{REFERENCIAS}

Benavides, J. D. P. (2018). Currículo oculto en la investigación formativa del Programa de Licenciatura en Educación Física. Lúdica Pedagógica, 1(28), Article 28. https://doi.org/10.17227/ludica.num28-9425.

Blondel, V. D., Guillaume, J.-L., Lambiotte, R., \& Lefebvre, E. (2008). Fast unfolding of communities in large networks. Journal of statistical mechanics: theory and experiment, 2008(10), P10008.

Bonchi, F., Castillo, C., Gionis, A., \& Jaimes, A. (2011). Social network analysis and mining for business applications. ACM Transactions on Intelligent Systems and Technology (TIST), 2(3), 1-37.

Dong, C., \& Rim, H. (2019). Exploring nonprofit-business partnerships on Twitter from a network perspective. Public relations review, 45(1), 104-118.

Fidalgo-Blanco, Á., \& Sein-Echaluce, M. L. (2018). Método MAIN para planificar, aplicar y divulgar la innovación educativa. Education in the Knowledge Society (EKS), 19(2), 83-101. https://doi.org/10.14201/eks201819283101.

García-Peñalvo, F. J. (2015). Mapa de tendencias en Innovación Educativa. Education in the Knowledge Society (EKS), 16(4), 6. https://doi.org/10.14201/eks2015164623

Ghinoi, S., \& Di Toma, P. (2021). Conceptualising business model innovation: Evidence from the managers' advice network. Innovation, 1-21.

Hernández Sampieri, R., Fernández Collado, C., \& Baptista Lucio, P. (2014). Metodología de la Investigación (6ta.). McGraw-Hill. 
Kuz, A., Falco, M., \& Giandini, R. (2016). Análisis de redes sociales: Un caso práctico. Computación y Sistemas, 20(1), 89-106.

Tabassum, S., Pereira, F. S. F., Fernandes, S., \& Gama, J. (2018). Social network analysis: An overview. WIREs Data Mining and Knowledge Discovery, 8(5), e1256. https://doi.org/10.1002/widm.1256.

Zhang, L., Guo, X., Lei, Z., \& Lim, M. K. (2019). Social network analysis of sustainable human resource management from the employee training's perspective. Sustainability, 11(2), 380.

\section{SEMBLANZA DEL AUTOR}

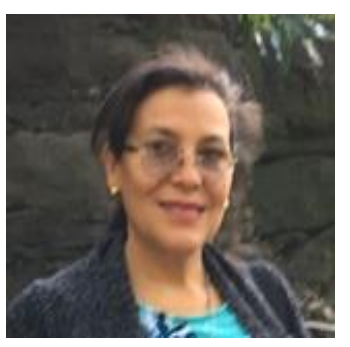

Patricia del C. Lacayo-Cruz: Se graduó de la carrera de Ingeniería en Computación de la Universidad Nacional de Ingeniería, Nicaragua, actualmente es profesor titular con más de 23 años de docencia y coordinadora I+D+i de la Facultad de Ciencias y Sistemas en la misma universidad. Además, posee una maestría en Informática Aplicada en los Sistemas de Gestión Empresarial y cursando la Maestría en Investigación Empresarial con el Instituto Tecnológico de Costa Rica, asimismo con experiencia en vice decanatura, gestora IPE, evaluadora curricular, consultora en Ingeniería de Software y tutoría de tesis monográfica. 